\title{
Towards a Robust Path Growing Algorithm for Semi-automatic MRI Segmentation
}

\author{
Casper F. Nielsen and Peter J. Passmore \\ School of Computing Science, Middlesex University, Bounds Green Road \\ London, N11 2NQ, United Kingdom \\ \{c.nielsen, p.passmore\}@mdx.ac.uk
}

\begin{abstract}
Segmentation of MRI volumes is complicated by RF inhomogeneity, noise and partial volume artifacts. Fully automatic methods often do not cope well with a combination of these problems. Semi-automatic methods are generally too simplistic or require a large amount of user interaction to work. Adaptable Class-Specific Representation (ACSR), implemented by the Path Growing Algorithm (PGA), is a semi-automatic segmentation framework, which has previously been demonstrated to produce accurate and robust segmentation of colour cryo section volumes with a minimal requirement for user interaction. This paper presents an evaluation of three different PGAs to implement ACSR for the MRI modality. Results are based on simulated data from the BrainWeb image database. Future work towards a robust solution is discussed.
\end{abstract}

\section{Introduction}

ACSR $[1,2]$ allows the user to define the goal of a segmentation visually by selecting representative class templates. The standard PGA, combined with Learning Vector Quantization (LVQ), has previously been used to implement robust ACSR segmentation of colour cryo section volumes [2] from the Visible Human Project. This study aims to bring the benefits of ACSR segmentation to the MRI modality.

\section{Evolving the PGA for MRI Segmentation}

A path in the PGA is a connected, acyclic chain of points where no point is repeated twice. All possible paths with distinct point sets are grown from a seed point via a $2 n$ connected expansion in $n$-dimensional space. All points in an image successively become the seed point and a path hierarchy for each class at every point is created by calculating the distance of each path to each class template and the path spread. Classspecific sampling windows are built from the winning paths and compete for the final classification. Boundary artefacts are eliminated or significantly reduced. To speed up ACSR segmentation the PGA may be combined with a preliminary crude segmentation step using LVQ, in which the PGA is applied only at boundary points (partial ACSR). See $[1,2,4]$ for a complete description and notation of the PGA. 
Due to less rich point descriptors in MRI compared to colour cryo section data we introduce path descriptors, constituted by the path median and the path average intensity difference between the seed point and all other points in the path. We consider three variations of the PGA with path descriptors: Sampling window built from a single path (PGA-SPD); sampling window built from a single path with the seed point shifted one point in the direction of growth for the calculation of path average intensity difference (PGA-SPDS) to achieve greater tolerance to noise; sampling window built from two full paths with shifted seed point (PGA-DPDS). In partial ACSR the LVQ segmented boundaries are dilated by a small factor and the PGA is applied the boundary points. The three new algorithms were tested with dilation factors of 1,3 and $\infty$ (full ACSR) on simulated T1 MRI volumes from the BrainWeb database (http://www.bic.mni.mcgill.ca/brainweb) with varying levels of RF inhomogeneity and noise (3\% noise, $20 \%$ inhomogeneity; $3 \%$ noise, $40 \%$ inhomogeneity; $7 \%$ noise, $20 \%$ inhomogeneity). Template selection was based on a simulated user in the form of the BrainWeb ground truth for the classes CSF, white matter and grey matter in eight slices per volume. Results were compared to previously published results by Dzung and Prince [3], where MRF segmentation was based on a standard Expectation Maximization (EM) algorithm and the Adaptive Generalized EM algorithm (AGEM).

Best results were found for PGA-SPDS with a dilation factor of 3, which was consistently better than the standard EM algorithm for all volumes and comparable to AGEM at the highest level of inhomogeneity and noise. Error rates (the ratio of misclassified pixels to the total number of pixels in the three segment classes) for this volume were $8.858 \%$ for PGA-SPDS, $10.699 \%$ for EM and $8.414 \%$ for AGEM.

\section{Conclusion}

We conclude that the single path representation with seed point shifting for path average intensity difference produced consistently better results. Partial ACSR not only speeded up the segmentation process but improved results, which were however affected by the lack of explicitly templated inhomogeneities. Future work will seek to reduce error rates through automated template creation at slice level from the initial LVQ segmentation. The work described in this paper is discussed in detail in [4].

\section{References}

1. Nielsen, C. F., Passmore, P. J: A Solution to the Problem of Segmentation Near Edges Using Adaptable Class-Specific Representation, Proc. 15th IEEE ICPR, (2000) 436-440

2. Nielsen, C. F., Passmore, P. J.: Achieving Accurate Colour Image Segmentation in 2D and 3D with LVQ Classifiers and Partial ACSR, Proc. Fifth IEEE WACV, (2000) 72-78

3. Dzung, L. P., Prince, J. L.: A Generalized EM Algorithm for Robust Segmentation of Magnetic Resonance Images, Proc. 33rd Ann. Conf. Inf. Sciences and Systems, (1999) 558-563

4. Nielsen, C. F., Passmore, P. J.: Towards a Robust Path Growing Algorithm for SemiAutomatic MRI Segmentation, Middlesex Uni. CS Tech. Report no. CS-01-01, (2001) 